\title{
Enhanced catecholamine transporter binding in the locus coeruleus of patients with early Parkinson disease
}

\author{
Ioannis U Isaias ${ }^{1,2,3^{*}}$, Giorgio Marotta ${ }^{4}$, Gianni Pezzoli ${ }^{2}$, Osama Sabri ${ }^{5}$, Johannes Schwarz ${ }^{3}$, Paolo Crenna ${ }^{1}$, \\ Joseph Classen ${ }^{3}$ and Paolo Cavallari ${ }^{1}$
}

\begin{abstract}
Background: Studies in animals suggest that the noradrenergic system arising from the locus coeruleus (LC) and dopaminergic pathways mutually influence each other. Little is known however, about the functional state of the LC in patients with Parkinson disease (PD).

Methods: We retrospectively reviewed clinical and imaging data of 94 subjects with PD at an early clinical stage (Hoehn and Yahr stage 1-2) who underwent single photon computed tomography imaging with FP-CIT $\left(\left[{ }^{123} \mid\right] \mathrm{N}-\omega^{-}\right.$

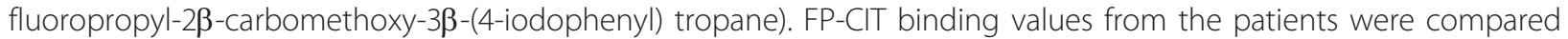
with 15 healthy subjects: using both a voxel-based whole brain analysis and a volume of interest analysis of a priori defined brain regions.

Results: Average FP-CIT binding in the putamen and caudate nucleus was significantly reduced in PD subjects (43\% and $57 \%$ on average, respectively; $p<0.001$ ). In contrast, subjects with PD showed an increased binding in the LC $(166 \%$ on average; $p<0.001)$ in both analyses. LC-binding correlated negatively with striatal FP-CIT binding values (caudate: contralateral, $\rho=-0.28, p<0.01$ and ipsilateral $\rho=-0.26, p<0.01$; putamen: contralateral, $\rho=$ $-0.29, p<0.01$ and ipsilateral $\rho=-0.29, p<0.01$ ).

Conclusions: These findings are consistent with an up-regulation of noradrenaline reuptake in the LC area of patients with early stage PD, compatible with enhanced noradrenaline release, and a compensating activity for degeneration of dopaminergic nigrostriatal projections.
\end{abstract}

\section{Background}

The pontine nucleus locus coeruleus (LC) is the major site of noradrenaline (NA) neurons in the central nervous system, hosting almost half of the NA-producing neurons in the brain [1].

The LC may play an important role in the pathophysiology of Parkinson disease (PD) for several reasons: (i) as a site of neuronal degeneration as part of PD pathology; [2] (ii) as the anatomical origin of projections modulating dopaminergic action of the substantia nigra; [3] (iii) as a structure under putative dopaminergic inhibitory control from the ventral tegmental area (VTA)

\footnotetext{
* Correspondence: ioannis.isaias@unimi.it

'Università degli Studi di Milano, Dipartimento di Fisiologia Umana, Milano, Italy

Full list of author information is available at the end of the article
}

which is known to degenerate in PD [4,5]. Based on physiological functions ascribed to the noradrenergic system, impaired functioning of LC in PD has been associated primarily to affective disorders, [6] cognitive disturbances, [7] sleep disorders, [8] sensory impairment [2] and autonomic dysfunction [9]. Through its interactions with the dopaminergic system however, the LC may also have a less direct role in the pathogenesis of PD via (i) an interplay of catecholamine systems with one amine cross-talking with receptors belonging to the other system $[10,11]$ or (ii) extra-synaptic neuro-modulatory, metabotroic and trophic activities of noradrenaline itself [12].

Information on the LC in PD is mainly based on postmortem examination of histopathological specimens, while information on its in vivo function is largely

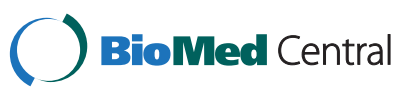

() 2011 Isaias et al; licensee BioMed Central Ltd. This is an Open Access article distributed under the terms of the Creative Commons Attribution License (http://creativecommons.org/licenses/by/2.0), which permits unrestricted use, distribution, and reproduction in any medium, provided the original work is properly cited. 
absent. Ideally, the LC-NA system and noradrenaline molecular transporters (NET) should be investigated in vivo by dedicated, highly specific radiotracers displaying low background non-NET binding, high sensitivity to variations in NET density and fast kinetics. As such a radiotracer is not available for large clinical studies, [13] we employed single photon computed tomography (SPECT) with FP-CIT ([ $\left.{ }^{123} \mathrm{I}\right] \mathrm{N}$ - $\omega$-fluoropropyl- $\beta$-carbomethoxy-3 $\beta$-(4-iodophenyl) tropane) in a large, homogeneous cohort of early stage PD patients. Although FPCIT is mainly used for assessing striatal dopamine reuptake transporters, it has shown sensitivity, albeit lower, to NET [14]. Therefore, when applied to an anatomical region with known low dopamine reuptake transporter capacity such as the LC, it allows investigation of the NA-dependent synaptic activity.

\section{Methods \\ Subjects}

We retrospectively reviewed clinical and imaging data of 94 subjects with idiopathic PD in whom FP-CIT SPECT was performed at the "Ospedale Maggiore Policlinico" in Milano within five years of the onset of motor symptoms. Fifteen healthy subjects (healthy controls, HC) were prospectively enrolled for comparisons of FP-CIT binding. At the time of SPECT, HC did not suffer from any disease and were not taking any medications. Clinical inclusion criteria for subjects with PD were: (a) diagnosis according to the UK Parkinson Disease Brain Bank criteria; (b) absence of any signs indicative for atypical parkinsonism (e.g. gaze abnormalities, autonomic dysfunction, significant psychiatric disturbances, etc.) over a follow-up period of at least three years after symptoms onset; (c) Hoehn and Yahr (H\&Y) stage 1 or 2 in drugs-off state (i.e. after overnight withdrawal of specific drugs for PD; no patients were taking long-acting dopaminergic drugs) at the time of SPECT; (d) positive clinical improvement at Unified Parkinson Disease Rating Scale (UPDRS) after L-Dopa intake (i.e. > 30\% from drug-off state) at some point during the three years of follow up; (e) a normal Magnetic Resonance Imaging (MRI) (no sign of white matter lesion or atrophy). Finally, given a putative role of LC and noradrenaline in cognition and mood (including depression) we excluded from this study patients with a positive score at UPDRS part I.

A quantitative profile of each patient' motor impairment was obtained from clinical assessment performed before SPECT by means of the UPDRS motor part (part III). L-Dopa daily dose and L-Dopa Equivalent Daily Doses (LEDDs) were also recorded, with the latter expressed as follows: $100 \mathrm{mg}$ levodopa $=1.5 \mathrm{mg}$ pramipexole $=6 \mathrm{mg}$ ropinirole. None of the subjects (both $\mathrm{PD}$ and $\mathrm{HC}$ ) were taking or stated to have ever been treated with antipsychotics or drugs known to affect the noradrenergic system (e.g., noradrenaline reuptake inhibitors). Drug naïve patients at the time of SPECT were not included in the study. The Ethics Committee of the Department of Human Physiology approved the study and all subjects gave informed consent.

\section{SPECT data acquisition and processing}

Intravenous administration of 110-140 MBq of FP-CIT (DaTSCAN, GE-Healthcare, UK) was performed 30-40 minutes after thyroid blockade (10-15 mg of Lugol oral solution) in the control subjects and in patients after overnight withdrawal of dopaminergic therapy [15]. Brain SPECT was performed 3 hours later by means of a dedicated triple detector gamma-camera (Prism 3000, Philips, Eindhoven, the Netherlands) equipped with lowenergy ultra-high resolution fan beam collimators ( 4 subsets of acquisitions, matrix size $128 \times 128$, radius of rotation 12.9-13.9 cm, continuous rotation, angular sampling: 3 degree, duration: 28 minutes). Brain sections were reconstructed with an iterative algorithm (OSEM, 4 iterations and 15 subsets) and then processed by 3D filtering (Butterworth, $5^{\text {th }}$ order, cut-off 0.31 pixel $^{-1}$ ) and attenuation correction (Chang method, factor 0.12).

\section{Imaging data analysis}

Two different and complementary image analyses were performed: a voxel-based whole brain analysis using Statistical Parametric Mapping SPM2 (Wellcome Department of Imaging Neuroscience, London, UK) implemented in MATLAB R2007a (The Mathworks Inc, USA), and a volume of interest (VOI) analysis of $a$ priori defined brain regions.

\section{SPM analysis}

A group-specific FP-CIT template was created by (i) spatially normalizing the FP-CIT images of 15 healthy subjects onto a FP-CIT MNI-based template, [16] (ii) subsequent averaging of the normalized images and their symmetric (mirror) images resulting in a mean image, and finally (iii) a smoothing of the mean image using a 3-dimensional Gaussian kernel with 8-mm full width at half maximum (FWHM). To increase the signal-to-noise ratio and account for subtle variations in anatomic structures, the individual subject's FP-CIT images were spatially normalized to this group-specific template and smoothed with a FWHM 10-mm Gaussian kernel. A reference region in the occipital cortex was defined as the union of the superior, middle and inferior occipital gyri along with the calcarine gyri VOIs defined by automated anatomical labelling (AAL), using the Wake Forest University (WFU) PickAtlas 2.4 software. Binding values for each subject's FP-CIT image were then computed in a voxel-by-voxel manner (voxel occipital reference)/(occipital reference). Using the 
General Linear Model in voxel-based statistical analysis of SPM2, a two-sample t-test contrast was used to elucidate group difference between PD and HC. No global normalization, or grand mean scaling, were applied, and the masking threshold was set to zero. Clusters of at least 35 voxels with the height threshold set at p < 0.001 , were considered significant.

\section{VOI analysis}

The LC FP-CIT binding values were for two VOIs (for left and right part of LC) created, using WFU Pick Atlas Tool, through the union of six distinct, contiguous Boxes (of $3 \mathrm{~mm}$ on the $z$ axis for each side), centered in the mean values on the $x$ and $y$ axis and dimensioned according to the standard deviation as proposed in Table 1 of Keren and coll., 2009 [17]. FP-CIT binding values for the caudate nucleus $(\mathrm{CN})$ and putamen $(\mathrm{PT})$ were calculated on the basis of VOIs defined with the Basal Ganglia Matching Tool [18]. Student's $t$-test was then applied. We defined as contralateral, those brain regions opposite to that of $\mathrm{PD}$ most severe sign presentation. For $\mathrm{HC}$, we referred to the right side as ipsilateral [15].

\section{General statistical analysis}

Unless otherwise stated, data are reported as median and range. Normality of data distribution was tested by means of Shapiro-Wilks test. Chi-Square was used to test gender distribution among groups. Demographic data were compared by means of Wilcoxon two-group test. The Spearman correlation coefficient was calculated to investigate statistical dependence among average binding values, demographic and clinical variables.

Table 1 Demographics and clinical data

\begin{tabular}{lcc}
\hline & PD & HC \\
\hline Subjects N. (male/female) & $94(67 / 27)$ & $15(4 / 11)^{*}$ \\
\hline Age at SPECT & $60(38-75)$ & $\begin{array}{c}63(51- \\
74)\end{array}$ \\
\hline Age at motor symptoms onset & $57(37-72)$ & \\
\hline Disease duration & $3(1-5)$ \\
\hline UPDRS motor score (part-III) [range 0 - & $19(8-56)$ \\
108] & $2(1-2)$ \\
\hline Hoehn and Yahr stage [range 1 - 5] & $400(0-850)$ \\
\hline L-Dopa in mg/day & $250(70-$ & \\
\hline LEDDs in mg/day & $1200)$ & \\
\hline
\end{tabular}

Data are reported as median and range (brackets). Age at SPECT, age at motor symptoms onset and disease duration are in years. All patients were evaluated with the Unified Parkinson Disease Rating Scale motor part (UPDRS part III) in drugs-off state (i.e. after overnight withdrawal of specific drugs for $\mathrm{PD}$, no patients was taking long-acting dopaminergic drugs). LEDDs were calculated as follow: $100 \mathrm{mg}$ levodopa $=1.5 \mathrm{mg}$ pramipexole $=6 \mathrm{mg}$ ropinirole. ${ }^{*} \mathrm{p}=0.0005$.
Statistical analyses were performed with the JMP statistical package, version 8.0 (SAS Institute, Inc., Cary, NC, USA).

\section{Results}

Table 1 shows the demographic and clinical characteristics of the study cohorts.

SPM analysis detected one large cluster of 6819 voxels (peaks at coordinates: $28-8-4$ and at $-31-8-4$ ) of significantly reduced FP-CIT binding involving the PT and the $\mathrm{CN}$ bilaterally (Figure 1, left). A cluster of 37 voxels (peak at coordinates: 2 -36 -26) with higher FP-CIT binding values was found in the LC of PD subjects (Figure 1 , right).

Volumes of interest analysis revealed reduced average binding values in the striatum and increased average binding value in LC area, bilaterally (Table 2).

FP-CIT binding in the striatum showed a weak, but significant, negative correlation with binding values of the corresponding LC (caudate: contralateral, $\rho=-0.28$, $\mathrm{p}=0.004$ and ipsilateral $\rho=-0.26, \mathrm{p}=0.008$; putamen: contralateral, $\rho=-0.29, \mathrm{p}=0.004$ and ipsilateral $\rho=$ -0.29, $\mathrm{p}=0.003$ ) (Figure 2). LC binding did not show other significant correlations. Finally, results for the FPCIT binding value in the LC area proved to be statistically independent when weighted for demographic (age at SPECT, age at onset, gender) and clinical characteristics (disease duration, disease severity and L-Dopa daily dose and LEDDs).

\section{Discussion}

\section{Increased FP-CIT binding in the LC area}

The present study provides in vivo evidence of higher baseline catecholamine transporter binding in the LC region in a large and homogeneous cohort of subjects with early PD. Our findings are consistent with an upregulation of noradrenaline reuptake in the LC area, which is well compatible with enhanced noradrenaline release $[19,20]$.

Our results are derived from the analysis of the binding of FP-CIT, a ${ }^{123}$ I-labeled cocaine derivative with high affinity for dopamine $\left(\mathrm{DAT} ; \mathrm{K}_{\mathrm{D}}=2 \mathrm{nM}\right)$ and a lesser affinity towards noradrenaline transporter $\left(\mathrm{NET} ; \mathrm{K}_{\mathrm{D}}\right.$ $=140 \mathrm{nM}$ ) [14]. Despite the higher affinity of FP-CIT for DATs, it is unlikely that the higher binding observed in the LC area is due to an enhanced dopaminergic, rather than noradrenergic, transporter for two main reasons: (i) in LC, DAT represent a minor and inconsistent component of the midbrain-derived dopaminergic terminals which degenerates in PD, along with other dopaminergic projections, [4] and (ii) in the LC a major NET component is synthesized in the cell body of pigmented neurons and exposed on their membrane to be transferred toward axonal terminals, [20] with a less 

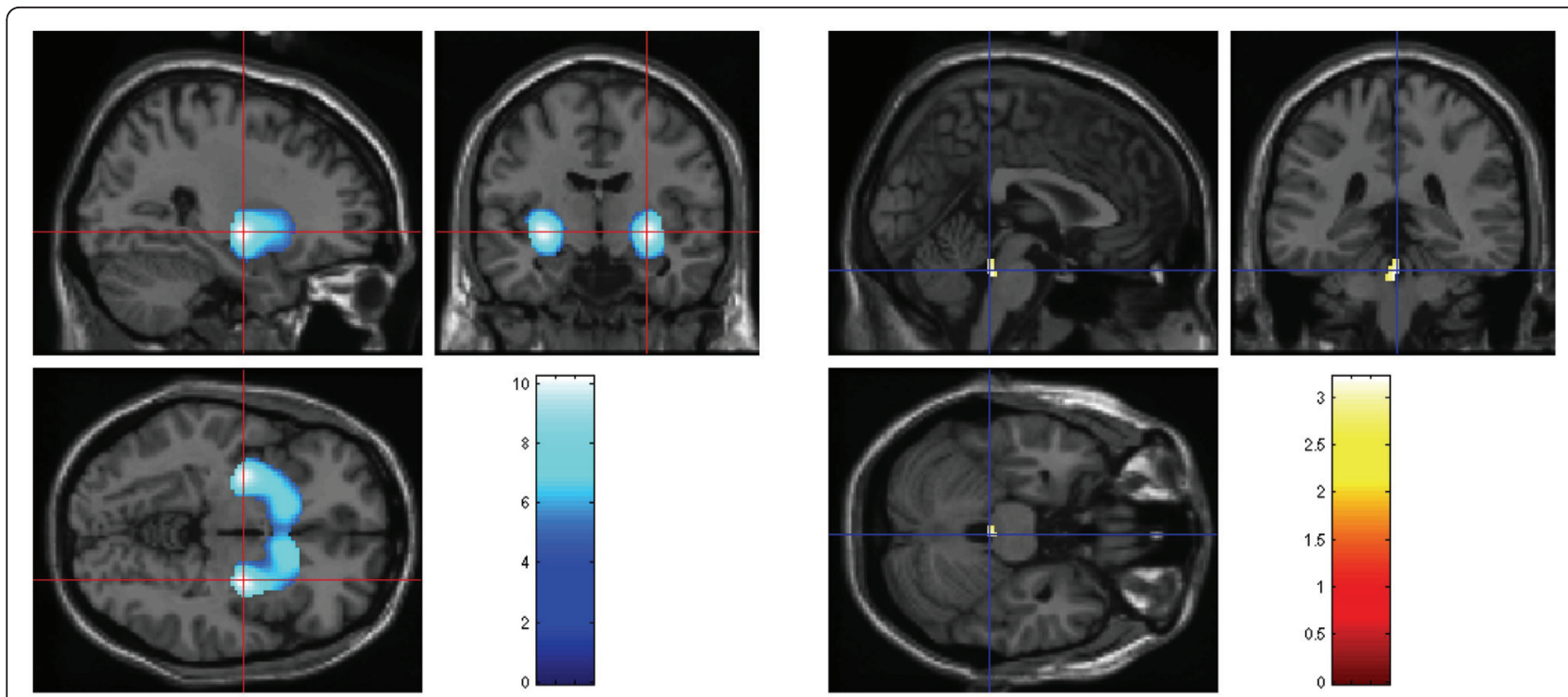

Figure 1 Overlay on a MRI showing the loss of FP-CIT binding bilaterally in the striatum (cluster of 6819 voxels, peaks at coordinates: $28-8-4$ and at $-31-8-4$ ) (left in the figure) and increased FP-CIT binding in the locus coeruleus area (cluster of 37 voxels, peak at coordinates: 2 -36 -26) (right in the figure) of the whole group of PD patients compared to controls.

consistent NET component localized on terminal projections arising from more caudal noradrenergic cell groups [21].

In $\mathrm{PD}$, reduced dopamine release from nigro-striatal projections results in loss and adaptive down-regulation of DAT binding sites in the striatal region [22]. In line with this notion, and in agreement with studies in de novo and early PD, where 40 to $60 \%$ of nigral dopaminergic neurons are lost, $[15,23]$ we found a significantly reduced FP-CIT binding in the caudate and putamen of PD patients. In contrast to the striatal compartment, analysis of FP-CIT labeling in the upper brainstem revealed significantly increased binding in a pontine area adjacent to the floor of the fourth ventricle and extending into the midbrain to the level of the inferior

Table 2 Binding values obtained with the analysis of volumes of interest

\begin{tabular}{lccc}
\hline Region of interest & PD & HC & p values \\
\hline CN contralateral & $3.18(1.2-3.84)$ & $5.27(3.51-6.15)$ & $<0.0001$ \\
\hline CN ipsilateral & $3.29(0.98-6.26)$ & $5.27(3.51-6.6)$ & $<0.0001$ \\
\hline PT contralateral & $1.86(0.65-4.72)$ & $4.83(3.07-6.04)$ & $<0.0001$ \\
\hline PT ipsilateral & $1.97(0.76-4.65)$ & $4.83(3.62-6.37)$ & $<0.0001$ \\
\hline LC contralateral & $313.5(81-663)$ & $131.43(59-371)$ & 0.001 \\
\hline LC ipsilateral & $321.17(87-632)$ & $123.6(38-354)$ & 0.0004
\end{tabular}

Data are reported as median and range (brackets). Average FP-CIT binding in the caudate nucleus (CN) and putamen (PT) was significantly reduced in subjects with PD subjects compared to HC. On the contrary, PD patients showed a significantly increased binding in the LC area (both right and left regions). We defined contralateral brain regions opposite to that of PD signs presentation. For $\mathrm{HC}$, we referred to the right side as ipsilateral [15]. colliculi. This area corresponds topographically to the LC coordinates identified by other studies including those employing neuromelanin-sensitive MRI methods $[6,17,24,25]$. In addition, the LC is the sole structure in the posterior rostral pons housing monoamine transporters [1], thus further supporting our claim of anatomical targeting of the LC.

Only two prior studies with PET have specifically investigated the LC in PD patients. A first, [24] reported a reduced ${ }^{18} \mathrm{~F}$-dopa intake in patients with advanced PD when compared to patients at an early stage of the disease. Because ${ }^{18} \mathrm{~F}$-dopa intake is more specifically related to dopaminergic neurotransmission, this study does not provide information on noradrenergic functioning of LC. In a second study, [6] PD subjects with depression showed a reduced binding of $\left[{ }^{11} \mathrm{C}\right] \mathrm{RTI}-32$, a marker of both DAT and NET, when compared to non-depressed patients. Interestingly, the noradrenergic activity of early non-depressed PD patients was within normal range in most patients and enhanced in few of them. In line with these findings, and having enrolled a larger and more selected cohort of subjects, we were able to reveal a significantly higher LC activity at an early stage of PD for the first time.

An acute effect of drugs on FP-CIT binding values appears unlikely since SPECT was performed after overnight withdrawal of anti-parkinsonian drugs. In addition, in animal studies, systemic administration of $\mathrm{D}_{2} / \mathrm{D}_{3}$ receptor agonists, such as pramipexole or apomorphine, showed little or no effect on the firing rate of LC-NA neurons [26]. Finally, a persistent treatment with 


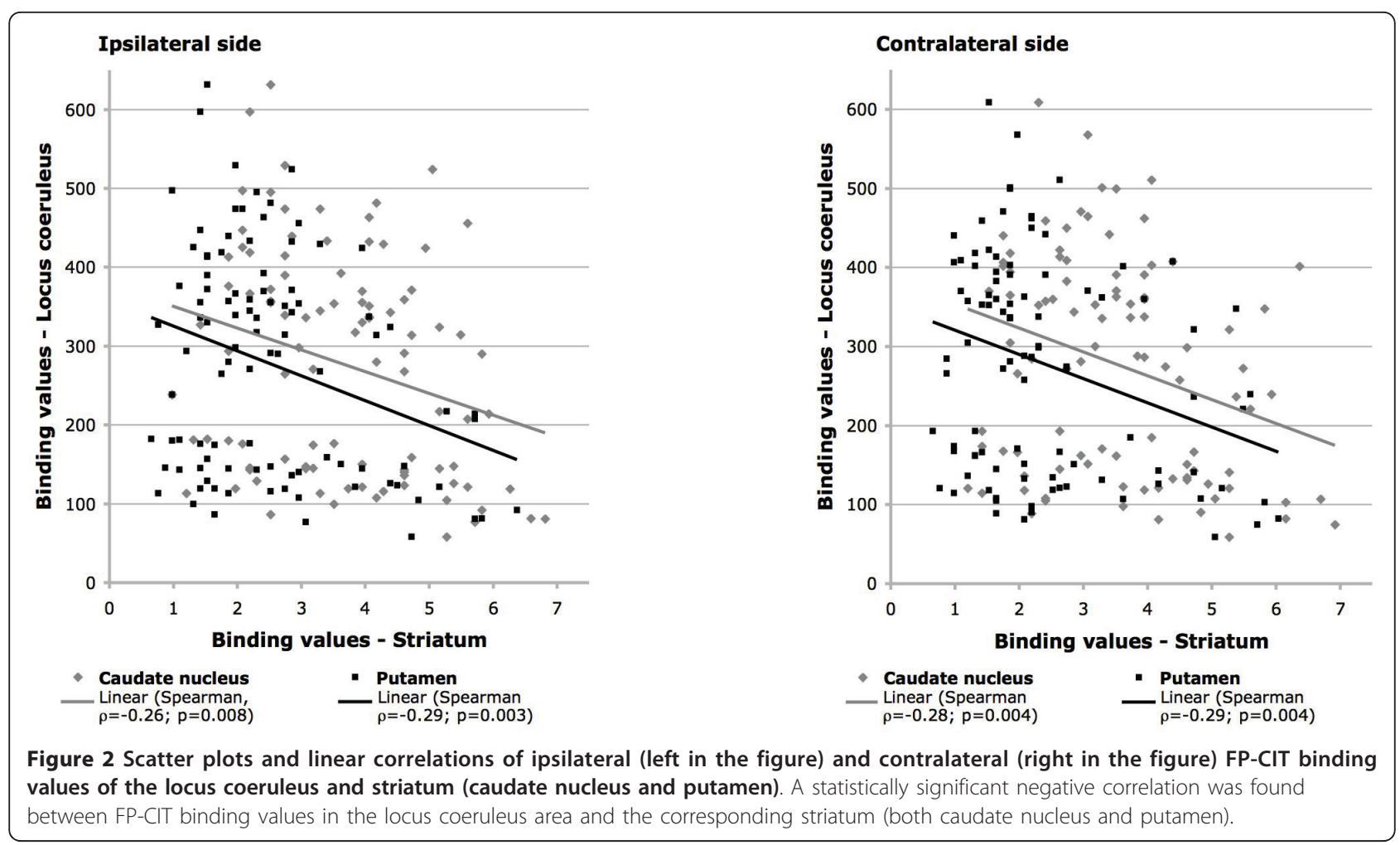

dopaminergic drugs will eventually down-regulate, rather than up-regulate, the surface expression of DAT and NET through internalization of the transporters $[27,28]$. Accordingly, the average FP-CIT binding values in the LC remained enhanced when data were L-Dopa weighted for equivalent daily dose and L-Dopa daily dose.

\section{In vivo versus anatomopathological studies}

Enhanced noradrenergic binding, and possibly activity, in PD might be considered at odds with neuropathological findings, where frank neuronal degeneration has been recognized within $\mathrm{LC}$, based on detection of specific cellular markers. Indeed, morphologic hallmarks of sporadic PD (Lewy bodies and dystrophic neurites containing pathologic $\alpha$-synuclein) may appear initially in the lower brainstem [2].

However, Lewy pathology can correlate poorly with neuronal loss in specific areas, thus its validity in predicting neuronal disintegration is questionable [29]. In fact, noradrenergic neurons in the LC are relatively preserved in early PD and do not exhibit the same intracellular changes as in the substantia nigra [30].

Accordingly, neuromelanin-sensitive imaging methods in vivo, [25] as well as anatomopathological studies suggested that the loss of NA neurons in PD may be confined to the larger, pigmented cells localized in the caudal part of the nucleus, whereas small unpigmented cells are increased in number, as if derived from shrinkage of larger neurons [31].

However, available information on the LC, so far derived from anatomopathological studies in subjects with $\mathrm{PD}$, is poorly comparable with our findings. In particular, the limited number of PD subjects investigated and the lack of clinical information (e.g. disease duration and the presence of depression or cognitive impairment) of patients in anatomopathological studies prevent a direct comparison between these studies and our results [31,32].

\section{Implications of enhanced LC-NA functioning in PD at an early stage}

Based on anatomical and histochemical data, along with neuropharmacological evidence, higher activity of the LC in PD may suggest: (i) in the striatum, noradrenaline plays a compensatory role cross targeting dopaminergic receptors (synaptic action); while (ii) in the substantia nigra, noradrenaline has a neuroprotective bolstering dopaminergic cells (extra-synaptic paracrine action).

As for the compensatory role, there is no absolute specificity for catecholaminergic substrate-receptor interactions, implying that one catecholamine can crosstalk with the pharmacologically defined receptors or transporters belonging to other catecholamines. Indeed, noradrenaline binds to pharmacologically defined dopaminergic receptors $[11,33,34]$. Therefore, enhanced 
noradrenaline release may be able to partially compensate a dopaminergic innervation loss due to degeneration of the substantia nigra.

With reference to a putative neuroprotective activity, noradrenaline suppresses pro-inflammatory and elevates anti-inflammatory molecules [35] and has the ability to scavenge superoxide and reactive oxygen species, which are thought to contribute to cellular damage and dopaminergic cell death [36]. Furthermore, the tottering mouse, which has noradrenergic hyperinnervation and increased levels of noradrenaline throughout the forebrain, appears to be protected from MPTP toxicity [37] while MPTP-induced damage to nigrostriatal dopaminergic neurons was potentiated by pretreatment with DSP-4, a selective LC neurotoxin [38]. Therefore, we speculate that enhanced LC-NA may be regarded as an endogenous paracrine agent promoting dopaminergic neuron survival $[39,40]$. This hypothesis would predict that degeneration of LC noradrenergic neurons in later stages of the disease might accelerate degeneration of substantia nigra dopaminergic neurons. The negative correlation between FP-CIT binding in the striatum and $\mathrm{LC}$ area is consistent with the above considerations of LC-NA compensatory and protective activity.

\section{Conclusions}

The present study suggests higher baseline catecholamine transporter binding in the LC area of patients with early stage PD. We propose that enhanced noradrenergic activity may be one factor modulating the severity of motor symptoms and may even influence progression of dopaminergic neurodegeneration.

\section{Acknowledgements}

The authors would like to thank Dr. Margherita Canesi, Dr. Swen Hesse, Dr. Philipp Meyer, Dr. Dorothee Saur and Dr. Paul Summers for their critical reading of the manuscript. The authors are grateful to Dr N. Tzourio-Mazoyer and colleagues for providing the AAL volumetric brain template, freely available at http://www.cyceron.fr/freeware.

The study was funded in part by a grant of the Grigioni Foundation for Parkinson disease.

\section{Author details \\ ${ }^{1}$ Università degli Studi di Milano, Dipartimento di Fisiologia Umana, Milano, Italy. ${ }^{2}$ Parkinson Institute, Istituti Clinici di Perfezionamento, Milano, Italy. ${ }^{3}$ Department of Neurology, University of Leipzig, Leipzig, Germany. ${ }^{4}$ Department of Nuclear Medicine, Fondazione IRCCS Ca' Granda - Ospedale Maggiore Policlinico, Milano, Italy. ${ }^{5}$ Department of Nuclear Medicine, University of Leipzig, Leipzig, Germany.}

\section{Authors' contributions}

IUI and GM participated in the conception of the study, gathered and analyzed the data. JC, PC, GP, OS, JS and PC contributed to data analysis and participated in the redaction of the paper. All authors read and approved the final manuscript.

\section{Competing interests}

The authors declare that they have no competing interests.
Received: 5 April 2011 Accepted: 21 July 2011 Published: 21 July 2011

\section{References}

1. Aston-Jones G, Shipley MT, Chouvet G, Ennis M, van Bockstaele E, Pieribone $V$ : Afferent regulation of locus coeruleus neurons: anatomy, physiology and pharmacology. Prog Brain Res 1991, 88:47-75.

2. Braak H, Del Tredici K, Rub U, de Vos RA, Jansen Steur EN, Braak E: Staging of brain pathology related to sporadic Parkinson's disease. Neurobiol Aging 2003, 24:197-211.

3. Collingridge $G L$, James TA, MacLeod NK: Neurochemical and electrophysiological evidence for a projection from the locus coeruleus to the substantia nigra. 1979, 290:44.

4. Javoy-Agid F, Agid Y: Is the mesocortical dopaminergic system involved in Parkinson disease? Neurology 1980, 30:1326-1330.

5. Guiard BP, El Mansari M, Blier P: Cross-talk between dopaminergic and noradrenergic systems in the rat ventral tegmental area, locus ceruleus, and dorsal hippocampus. Mol Pharmacol 2008, 74:1463-1475.

6. Remy P, Doder M, Lees A, Turjanski N, Brooks D: Depression in Parkinson's disease: loss of dopamine and noradrenaline innervation in the limbic system. Brain 2005, 128:1314-1322.

7. Zweig RM, Cardillo JE, Cohen M, Giere S, Hedreen JC: The locus ceruleus and dementia in Parkinson's disease. Neurology 1993, 43:986-991.

8. Boeve BF, Silber MH, Saper CB, Ferman TJ, Dickson DW, Parisi J: Pathophysiology of REM sleep behaviour disorder and relevance to neurodegenerative disease. Brain 2007, 130:2770-2788.

9. Ørskov L, Jakobsen J, Dupont E, de Fine Olivarius B, Christensen NJ: Autonomic function in Parkinsonian patients relates to duration of disease. Neurology 1987, 37:1173-1178.

10. Devoto P, Flore G: On the origin of cortical dopamine. Is it a cotransmitter in noradrenergic neurons? Curr Neuropharmacol 2006, 4:115-125

11. Cornil CA, Ball GF: Interplay among Catecholamine Systems: Dopamine Binds to $a_{2}$-Adrenergic Receptors in Birds and Mammals. J comp neurol 2008, 511:610-627.

12. Waterhouse BD, Devilbiss D, Fleischer D, Sessler FM, Simpson KL: New perspectives on the functional organization and postsynaptic influences of the locus ceruleus efferent projection system. Adv Pharmacol 1998, 42:749-754.

13. Logan J, Wang G, Telang F, Fowler JS, Alexoff D, Zabroski J: Imaging the norepinephrine transporter in humans with $(S, S)-\left[{ }^{11} \mathrm{C}\right] \mathrm{O}$-methyl reboxetine and PET: problems and progress. NuC Med Biol 2007, 34:667-679.

14. Booij J, Kemp P: Dopamine transporter imaging with [ ${ }^{123}$ I]FP-CIT SPECT: potential effects of drugs. Eur J Nucl Med Mol Imaging 2008, 35:424-438.

15. Isaias IU, Benti R, Cilia R, Canesi M, Marotta G, Gerundini P, Pezzoli G, Antonini A: [123l]FP-CIT striatal binding in early Parkinson's disease patients with tremor vs. akinetic-rigid onset. Neuroreport 2007, 14:1499-1502.

16. Ma Y, Dhawan V, Mentis M, Chaly T, Spetsieris PG, Eidelberg D: Parametric mapping of [18F]FPCIT binding in early stage Parkinson's disease: a PET study. Synapse 2002, 45:125-133.

17. Keren NI, Lozar CT, Harris KC, Morgan PS, Eckert MA: In vivo mapping of the human locus coeruleus. Neurolmage 2009, 47:1261-1267.

18. Calvini P, Rodriguez G, Inguglia F, Mignone A, Guerra UP, Nobili F: The basal ganglia matching tools package for striatal uptake semiquantification: description and validation. Eur J Nuc Med Mol Imaging 2007, 34:1240-1253.

19. Metzger RR, Brown JM, Sandoval V, Rau KS, Elwan MA, Miller GW: Inhibitory effect of reserpine on dopamine transporter function. Eur J Pharmacol 2002, 456:39-43.

20. Zahniser NR, Sorkin A: Trafficking of dopamine transporters in psychostimulant actions. Semin Cell Dev Biol 2009, 20:411-417.

21. Ordway GA, Stockmeier CA, Cason GW, Klimek V: Pharmacology and Distribution of Norepinephrine Transporters in the Human Locus Coeruleus and Raphe Nuclei. Journal Neurosci 1997, 17:1710-1719.

22. Lee CS, Samii A, Sossi V, Ruth TJ, Schulzer M, Holden JE: In vivo positron emission tomographic evidence for compensatory changes in presynaptic dopaminergic nerve terminals in Parkinson's disease. Ann Neurol 2000, 47:493-503.

23. Brooks DJ: The early diagnosis of Parkinson's disease. Ann Neurol 1998 44(Suppl 1):10-18. 
24. Moore RY, Whone AL, Brooks DJ: Extrastriatal monoamine neuron function in Parkinson's disease: An ${ }^{18} \mathrm{~F}$-dopa PET study. Neurobiol of Dis 2008, 29:381-390.

25. Sasaki M, Shibata E, Tohyama K, Takahashi J, Otsuka K, Tsuchiya K: Neuromelanin magnetic resonance imaging of locus ceruleus and substantia nigra in Parkinson's disease. Neuroreport 2006, 17:1215-1218.

26. Chernoloz O, Mansari ME, Blier P: Sustained administration of pramipexole modifies the spontaneous firing rate of dopamine, norepinephrine, and serotonin neurons in the rat brain. Neuropsychopharmacology 2009, 34:651-661.

27. Saunders C, Ferrer JV, Shi L, Chen J, Merrill G, Lamb ME: Amphetamineinduced loss of human dopamine transporter activity: an internalizationdependent and cocaine-sensitive mechanism. Proc Natl Acad Sci USA 2000, 97:6850-6855.

28. Kahlig KM, Javitch JA, Galli A: Amphetamine regulation of dopamine transport. Combined measurements of transporter currents and transporter imaging suport the endocytosis of an active carrier. J Biol Chem 2004, 279:8966-8975.

29. Jellinger KA: Formation and development of Lewy pathology: a critical update. J Neurol 2009, 256(Suppl 3):270-279.

30. Halliday GM, Ophof A, Broe M, Jensen PH, Kettle E, Fedorow H: a-Synuclein redistributes to neuromelanin lipid in the substantia nigra early in Parkinson's disease. Brain 2005, 128:2654-2664.

31. Hoogendijk WJ, Pool CW, Troost D, van Zwieten E, Swaab DF: Image analyser-assisted morphometry of the locus coeruleus in Alzheimer's disease, Parkinson's disease and amyotrophic lateral sclerosis. Brain 1995, 118:131-43.

32. McMillan PJ, White SS, Franklin A, Greenupa JL, Leverenz JB, Raskind MA, Szot $P$ : Differential response of the central noradrenergic nervous system to the loss of locus coeruleus neurons in Parkinson's disease and Alzheimer's disease. Brain Res 2011, 1373:240-252.

33. Cornil CA, Balthazart J, Motte P, Massotte L, Seutin V: Dopamine activates noradrenergic receptors in the preoptic area. J Neurosci 2002, 22:9320-9330.

34. Newman-Tancredi A, Audinot-Bouchez V, Gobert A, Millan MJ: Noradrenaline and adrenaline are high affinity agonists at dopamine $D_{4}$ receptors. Eur J Pharmacol 1997, 319:379-383.

35. Feinstein DL, Heneka MT, Gavrilyuk V, Dello Russo C, Weinberg G, Galea E: Noradrenergic regulation of inflammatory gene expression in brain. Neurochem Int 2002, 41:357-365.

36. Traver S, Salthun-Lassalle B, Marien M, Hirsch EC, Colpaert F, Michel PP: The neurotransmitter noradrenaline rescues septal cholinergic neurons in culture from degeneration caused by low-level oxidative stress. $\mathrm{Mol}$ Pharmacol 2005, 67:1882-91.

37. Frey K, Kilbourn M, Robinson T: Reduced striatal vesicular monoamine transporters after neurotoxic but not after behavioral-sensitizing doses of methamphetamine. Eur J Pharmacol 1997, 334:273-279.

38. Mavridis M, Degryse AD, Lategan AJ, Marien MR, Colpaert FC: Effects of locus coeruleus on parkinsonian signs, striatal dopamine and substantia nigra cel loss after 1-methyl-4-phenyl-1,2,3,6-tetrahydropyridine monkeys: a possible role for the locus coeruleus in progression of Parkinson's disease. Neuroscience 1991, 41:507-523.

39. Rommelfanger KS, Weinshenker D: Norepinephrine: the redheaded stepchild of Parkinson disease. Biochem Pharmacol 2007, 74:177-190.

40. Berglöf $E$, Strömberg I: Locus coeruleus promotes survival of dopamine neurons in ventral mesencephalon. An in oculo grafting study. Exp Neurol 2009, 216:158-65.

\section{Pre-publication history}

The pre-publication history for this paper can be accessed here: http://www.biomedcentral.com/1471-2377/11/88/prepub

doi:10.1186/1471-2377-11-88

Cite this article as: Isaias et al:: Enhanced catecholamine transporter binding in the locus coeruleus of patients with early Parkinson disease. BMC Neurology 2011 11:88. 